\title{
Surface-Emitting Perovskite Random Lasers for Speckle-Free Imaging
}

Yilin Liu ${ }^{\dagger}$, Wenhong Yang ${ }^{\dagger}$, Shumin Xiao ${ }^{\dagger, t}, \#$, Nan Zhang ${ }^{\dagger}$, Yubin Fan ${ }^{\dagger}$, Geyang Qu ${ }^{\dagger}$, Qinghai Song ${ }^{\dagger, t}, *$

$\dagger$ State Key Laboratory on Tunable laser Technology, Ministry of Industry and Information Technology Key Lab of Micro-Nano Optoelectronic Information System, Shenzhen Graduate School, Harbin Institute of Technology, Shenzhen, 518055, China.

\$Collaborative Innovation Center of Extreme Optics, Shanxi University, Taiyuan 030006 China

\# shumin.xiao@hit.edu.cn; * qinghai.song@hit.edu.cn; 


\section{The properties of lead halide perovskite films}

A

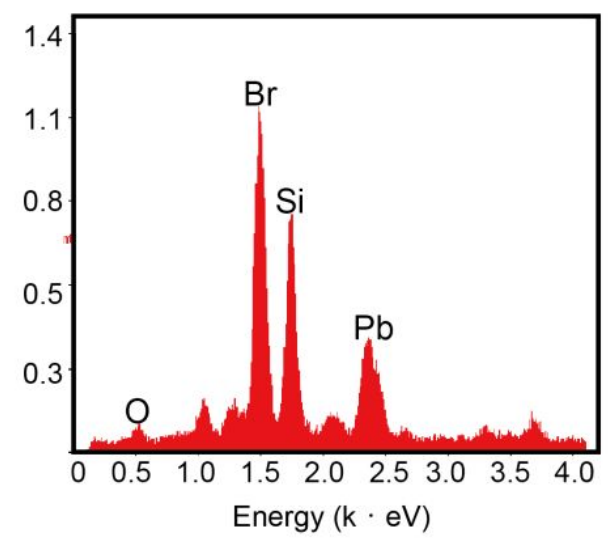

C

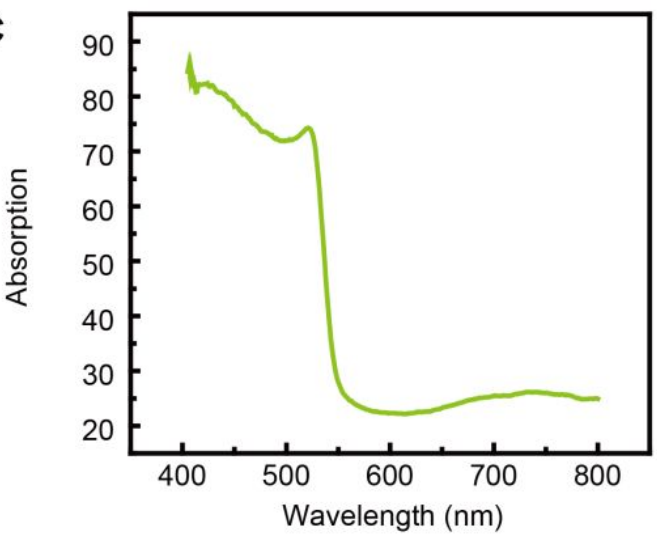

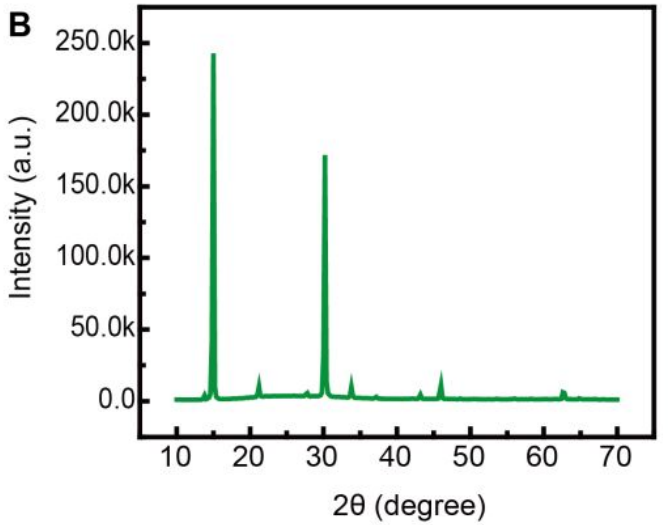

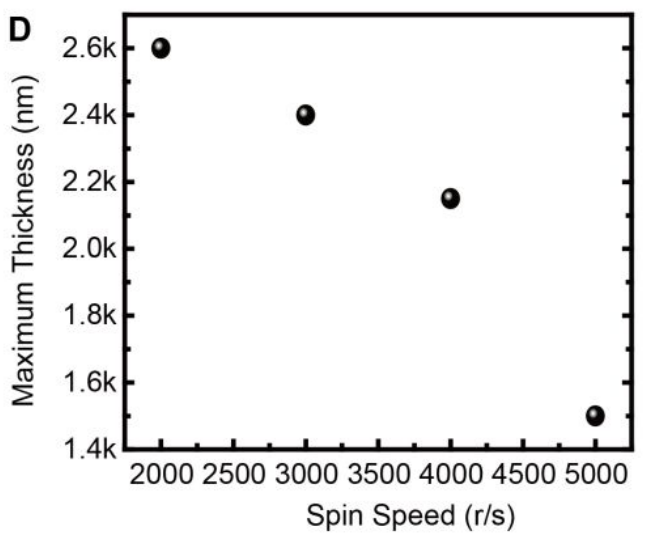

Figure S1. The physical and chemical characterization of perovskite films. (A) The energy dispersive X-Ray spectroscopy of the perovskite films. (B) The X-ray diffraction spectrum of the films. (C) The absorption spectrum of the films. (D) The thickness of this film at different spin-coating speed.

In this main text, we have mentioned the properties of lead halide perovskite films. Here we show the experimental details. Figure S1A shows that the ratio of $\mathrm{Br}: \mathrm{Pb}$ is around 3, which matches the $\mathrm{MAPbBr}_{3}$ very well. The corresponding X-ray diffraction spectrum is shown in Figure S1B. Two dominant peaks match the previous reports very well. Similarly, we have also measured the absorption of the perovskite 
film. A clear bandedge can be seen at $\sim 2.25 \mathrm{eV}$ in Figure S1C. At last, we notice that the perovskite film is not uniform and smooth. It is composed of numerous perovskite islands. Figure S1D summarizes the averaged maximal heights of these islands as a function of spin-coating speed. It is easy to see that the thickness decreases with the increase of spin-coating speed. This information is important to the numerical fitting of surface emitting random lasers (SERLs) in the main manuscript.

\section{Optical measurements}

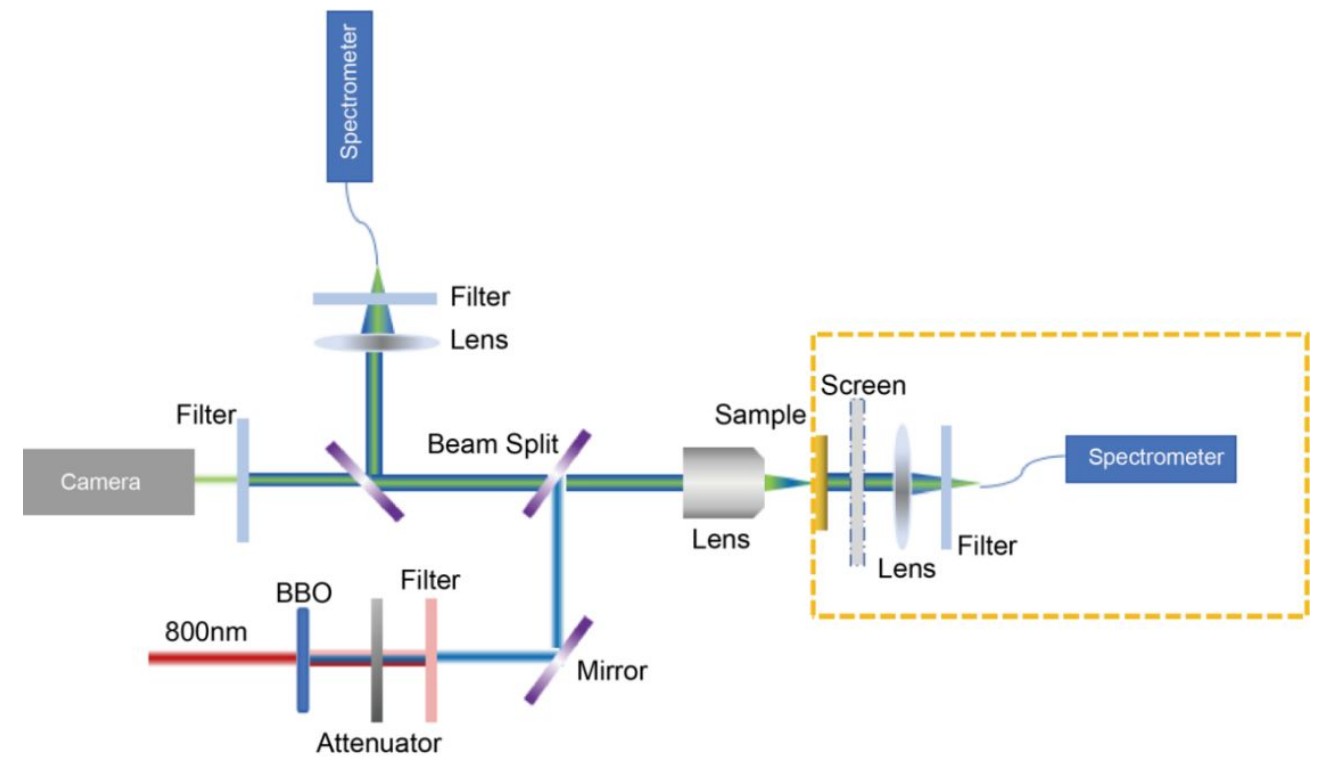

Figure S2. The optical setup for lasing and imaging experiments of perovskite random laser.

In the main manuscript, we have studied the laser properties of lead halide perovskite based SERLs and random lasers. The details of optical setup are shown in Fig. S2. A 800nm femtosecond Ti:Sapphire laser beam was lasing from a regenerative amplifier $(800 \mathrm{~nm}$, repetition rate $1 \mathrm{kHz}$, pulse width 100 fs, seeded by MaiTai, Spectra Physics) . A BBO ( $\beta$-barium borate) crystal was used to frequency doubling this laser beam to $400 \mathrm{~nm}$. Behind the $\mathrm{BBO}$, a band-pass filter centered around 400 
$\mathrm{nm}$ was placed to leach out the rest idle light $(800 \mathrm{~nm})$. Then the pump light was entered into a homemade micro-photoluminescence microscope and focused on the top surface of perovskite films by a $40 \times$ objective lens. The emitted light from the perovskite samples was collected by the same objective lens, and was collected with a lens to the CCD coupled spectrometer via a multimode fiber after filtered the pump light. The measurement of SERLs was using the transmission light path as shown in the orange dotted box, and the objective lens was changed to a $5 \times$ one. The screen in the box was used to show the far field patterns of the sample. The other parts were used as described above.

\section{Random lasers in a single perovskite grain}
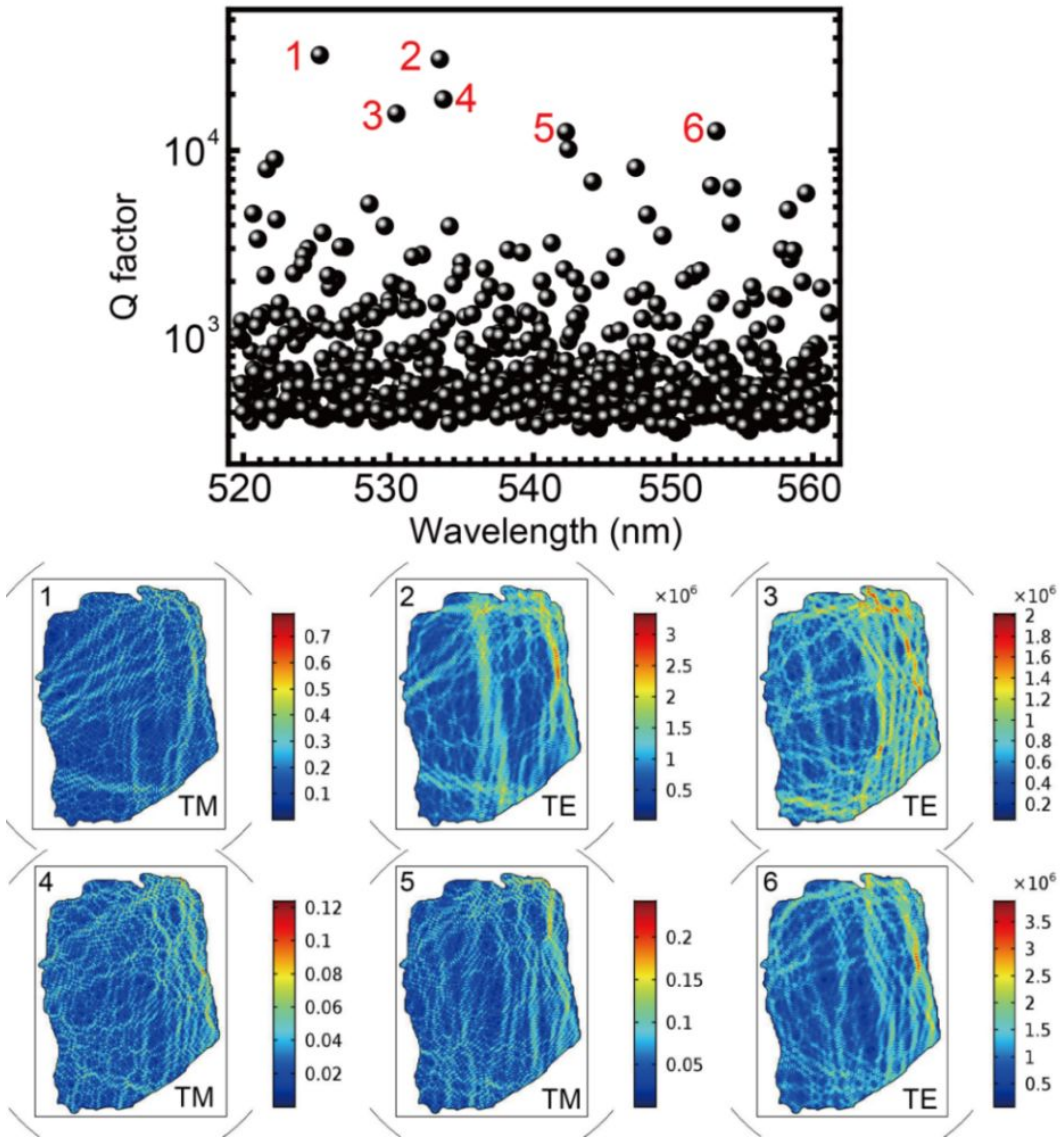

Figure S3. The numerical simulated resonances and the corresponding field patterns 
of modes 1-6 marked in top-panel.

The random lasers in a single perovskite grain are also very interesting. Different from the random lasers in condensed nanoparticles, here the multiple scatterings are formed by the rough cavity boundary and are partially assisted by the total internal reflection. Fig. S3 shows the numerical results of the resonant modes in a single perovskite grain. All the refractive index and light extinction coefficient are measured by the ellipsometer. In our numerical calculation, the two-dimensional cavity is simulated. The cavity boundary shape is taken from the top-view scanning electron microscope (SEM) image. But the cavity size is reduced to $1 / 5$ of the real size to save the computer resources and time. The top panel of Fig. S3 shows the Q factors of resonant modes within the perovskite grain. Numerous resonant modes have been obtained. Due to the assistance of total internal reflection, there are some modes can have $\mathrm{Q}$ factors above $10^{4}$. However, the $\mathrm{Q}$ factors of these modes are very close and much lower than the conventional whispering gallery modes. Meanwhile, the resonant wavelengths are quite random. The bottom panels show the field patterns of relatively high-Q resonant modes marked by 1-6 in the top panel. We can see that their field distributions are exactly quite random and different from one another. This means that these random modes are formed by modes with different transverse orders instead of the longitude orders. In this sense, the random lasers are ideal lasing sources for low and controllable spatial coherence. 


\section{The reduced speckle pattern with low spatial coherence perovskite}

\section{random lasers}

A

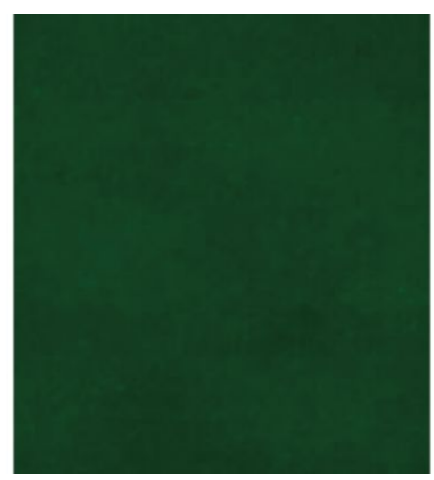

B

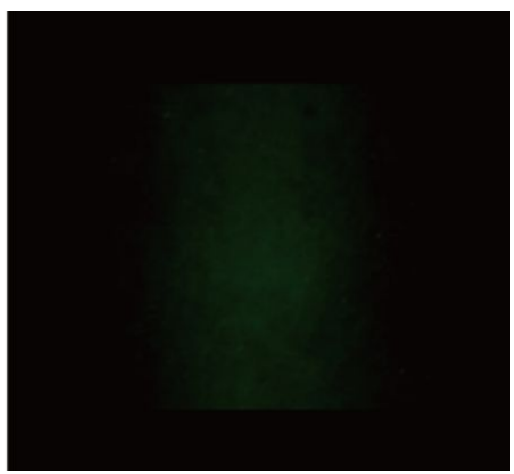

Figure S4. The spatial coherence and speckle patterns. (A) The speckle patterns of the perovskite film random laser. (B) The spatial coherence $\gamma$ of the random lasers in perovskite film without the DBR mirrors.

In the main manuscript, we have studied the random lasers in a perovskite films without applying the distributed Bragg reflectors (DBRs). In additional to the random laser spectrum and threshold behaviors, we have also studied their applications in speckle free imaging. The optical setup is the same as the top-panel in Fig. 4a in the main manuscript. Figure S4A shows the image taken by the CCD camera after the random lasers pass through a diffusive glass slide. Different from the laser diode, the image is quite uniform with negligible variations, giving a spatial contrast $\mathrm{C}=0.0289$. This kind of low speckle contrast is attributed to the low spatial coherence of the random lasers, which are measured by double slit interference. The experimental results are summarized in Fig. S4B. Almost no interference pattern can be seen and 
the calculated spatial coherence is about $\gamma=0.11$.

\section{The design of SERL}

A

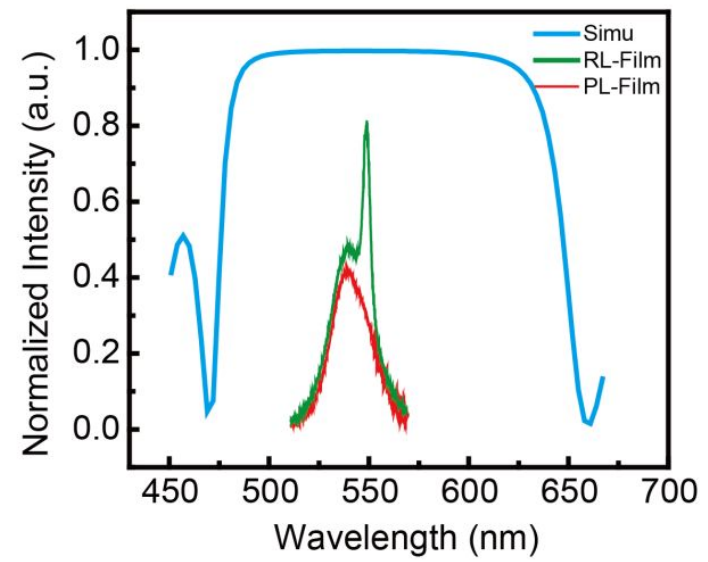

B

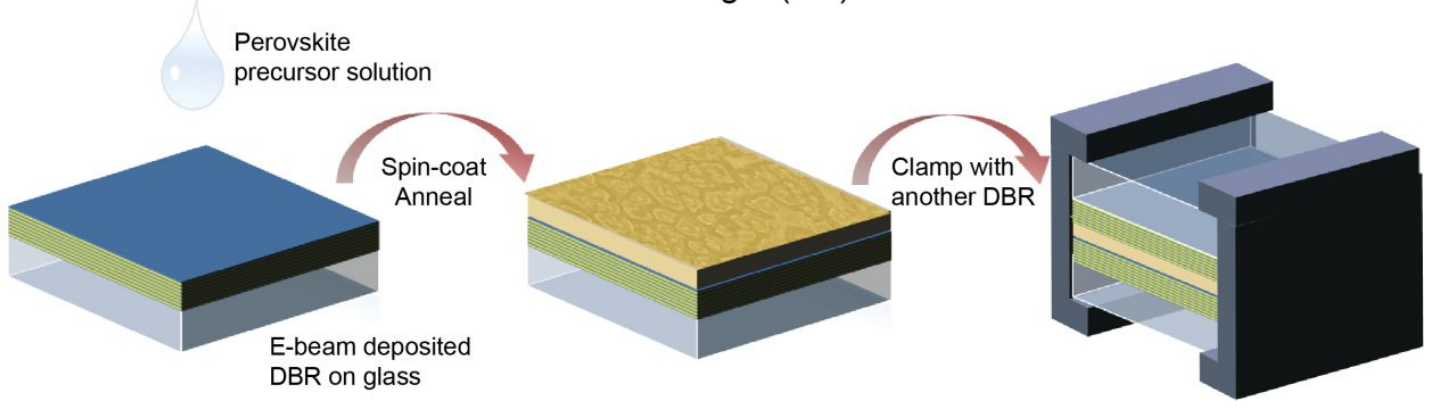

Figure S5. Design and preparation process of the DBR-Perovskite-DBR device. (A)

Reflectance spectrum of designed multilayer structure (blue line). The spectrum of perovskite film random laser (green line), and photoluminescence spectrum (orange line) The high reflective region $(480 \mathrm{~nm}-630 \mathrm{~nm})$ covering the lasing region of the perovskite film completely. (B) Schematic of preparation process, the preparation of the perovskite film was a simple one-step method. More detailed information was showed in Method.

In principle, the SERL is composed of two essential parts, i.e. the transverse random lasers and the vertical planar microcavity. The first one is controlled by the 
gain medium and the scattering, whereas the latter one is determined by the thicknesses of $\mathrm{TiO}_{2} / \mathrm{SiO}_{2}$ films. Therefore, the design of SERL can be simplified to the following steps.

1. Measuring the gain spectrum and laser spectrum. This is the step to confirm the generation of random laser and the operation wavelength.

2. Numerically calculating the reflection spectrum of DBR mirrors. This can be done with either transfer matrix or a commercial software (COMSOL Multiphysics). The key factor is determining the thicknesses and make the refraction at the lasing wavelength above $95 \%$.

3. Spin-coating the disorder perovskite film onto the DBR and covering it with another DBR mirror. This is the final step to realize the vertical planar microcavity.

To make the design of SERL clearly, we have plotted the schematic illustration and added the corresponding transfer matrix. As mentioned above, the reflection or transmission spectrum of a DBR or a planar microcavity can be calculated with either a COMSOL Multiphysics or a transfer matrix. The COMSOL Multiphysics is a commercial software, which is not stated here. We briefly show the transfer matrix below. Basically, the matrix representing light propagation through a period of films in vertical direction. 


$$
M=\left[\begin{array}{cc}
\cos \delta & \frac{i \sin \delta}{n} \\
i n \sin \delta & \cos \delta
\end{array}\right]
$$

$\delta=(2 \pi / \lambda) h$, in which $\mathrm{h}$ is the thickness of the film, and $\mathrm{n}$ is is the refractive index. $\mathrm{h}_{\mathrm{TiO} 2}=60 \mathrm{~nm}, \mathrm{~h}_{\mathrm{SiO} 2}=95 \mathrm{~nm}$ and $\mathrm{n}_{\mathrm{TiO} 2}=2.2, \mathrm{n}_{\mathrm{SiO} 2}=1.47$. Film thickness was from experimental results. The transmission matrix of the whole cavity is:

$$
M^{n}=M_{\mathrm{SiO} 2} M_{\mathrm{TiO} 2} \ldots M_{\mathrm{SiO} 2} M_{\mathrm{TiO} 2} M_{\text {air }} M_{\mathrm{PerO}} M_{\mathrm{TiO}_{2}} M_{\mathrm{SiO} 2} \ldots M_{\mathrm{TiO} 2} M_{\mathrm{SiO} 2}
$$

$M_{\mathrm{SiO} 2}, M_{\mathrm{TiO} 2}, M_{\mathrm{Pr}}$ are transmission matrixes of $\mathrm{SiO}_{2}, \mathrm{TiO}_{2}$ and perovskite. Reflectance at wavelength $\lambda$ is

$$
r=\frac{\left[\left(m_{11}^{n}+m_{12}^{n} n_{s}\right) n_{0}-\left(m_{21}^{n}+m_{22}^{n} n_{s}\right)\right]}{\left[\left(m_{11}^{n}+m_{12}^{n} n_{s}\right) n_{0}+\left(m_{21}^{n}+m_{22}^{n} n_{s}\right)\right]}
$$

where $m_{11}^{n}, m_{12}^{n}, m_{21}^{n}, m_{22}^{n}$ are matrix elements of $M^{n}, n_{0}=1$ and $n_{s}=1.46$ indicate the reflective index of air and substrate, respectively.

\section{Effect of scattering on reflection resonance}




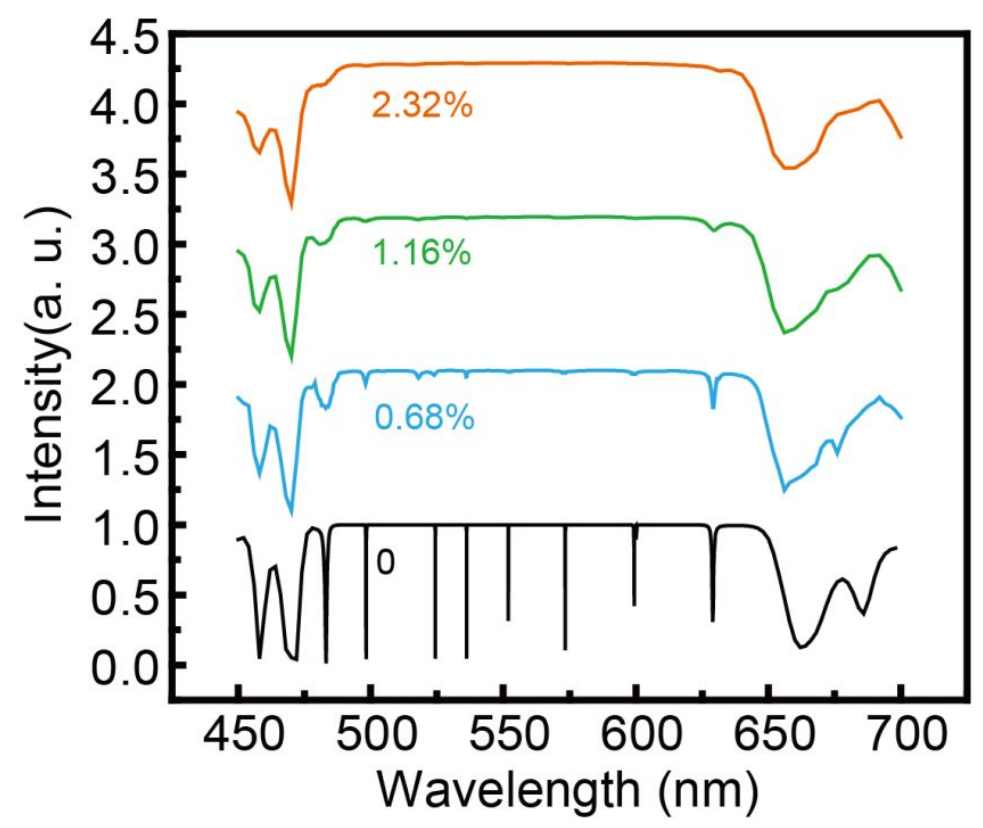

Figure. S6 The reflectance of DBR-Perovskite-DBR cavity with scattered pheres of different densities.

We note that the scattering plays an important role in the reflection spectrum in experiment, making the resonance shallower and broader. This information can be clearly seen from Fig. S6. When the planar microcavity is absent of scattering medium, here are several dips in the high reflectance region of this device. Then nanoparticles have been added in the perovskite film in model. The reflective index of the spheres is 1.5 , which is lower than the perovskite and thus can induce strong scattering. As shown in Fig. S6, the resonant dips become increasingly less obvious with the increase of the density of scattering spheres in film. This result shows that the strong scattering from the rough perovskite films sandwiched between the DBRs would make resonant peaks become shallow under decoherence. It also explains the reason that the resonant dips are shallower in experimentally recorded reflection spectrum. 


\section{The reflection spectrum of the DBR mirrors}

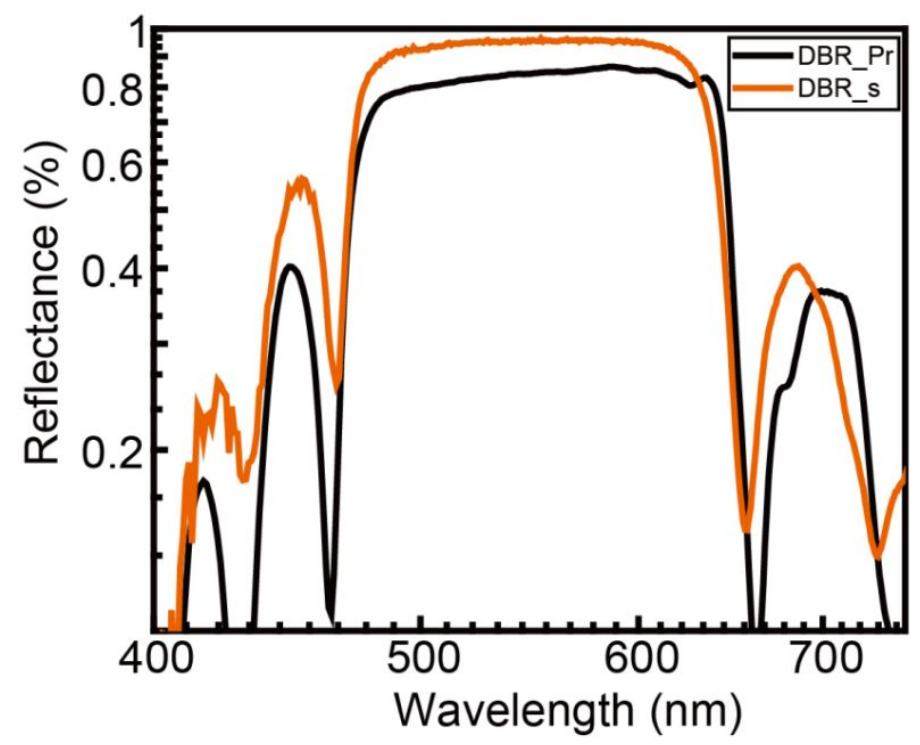

Figure S7. The comparison of the reflectivity between a single DBR and the micro-cavity.

In the main manuscript, we have deposited pairs of $\mathrm{TiO}_{2} / \mathrm{SiO}_{2}$ films and achieved the DBR mirrors. Here we show the experimentally recorded reflection spectrum of the DBR mirrors. The results are shown in Fig. S7 below. The orange line in Fig. S7 shows the reflection spectrum of DBR. The reflectance is over $90 \%$ in a broad spectral range from $476 \mathrm{~nm}$ to $648 \mathrm{~nm}$. For a direct comparison, we have also plotted the reflection spectrum of SERL microcavity (black line in Fig. S7). Due to the presence of scattering loss of perovskite film, the reflectance of a SERL cavity is about $10 \%$ lower than a single DBR mirror. Meanwhile, as mentioned in the main text, the resonant dips are hardly to be seen either. This is different from conventional planar microcavity and is also caused by the strong scattering loss of perovskite film.

\section{Far field patterns of SERLs}




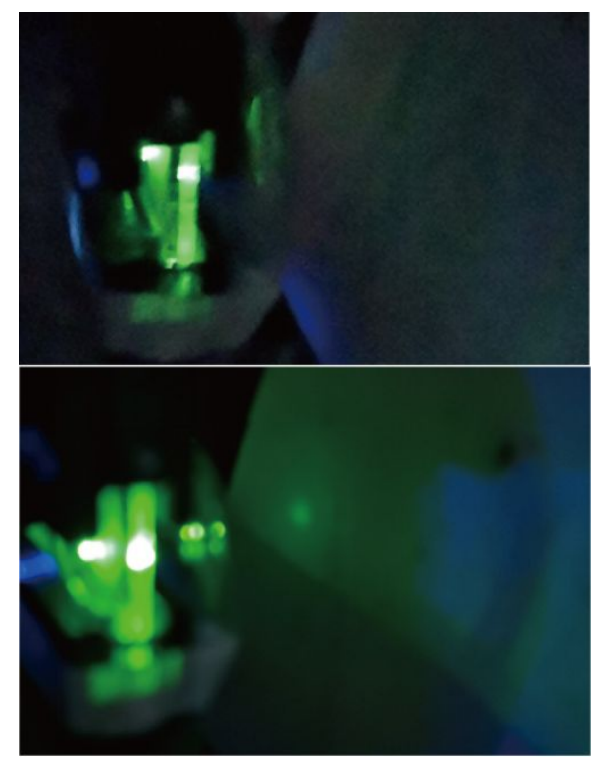

Figure S8. The far field image of the emission below and over threshold.

The high directionality is an essential characteristic of SERL in this manuscript. Here we show that the highly directional emission is caused by the laser instead of the nanostructures. If the nanostructure itself can produce directional output, it should also be observed at below threshold. As shown in Fig. S8 below, the highly directional laser beam can only be observed when the pumping power is above the threshold.

\section{The lasing photostability of the SERLs}
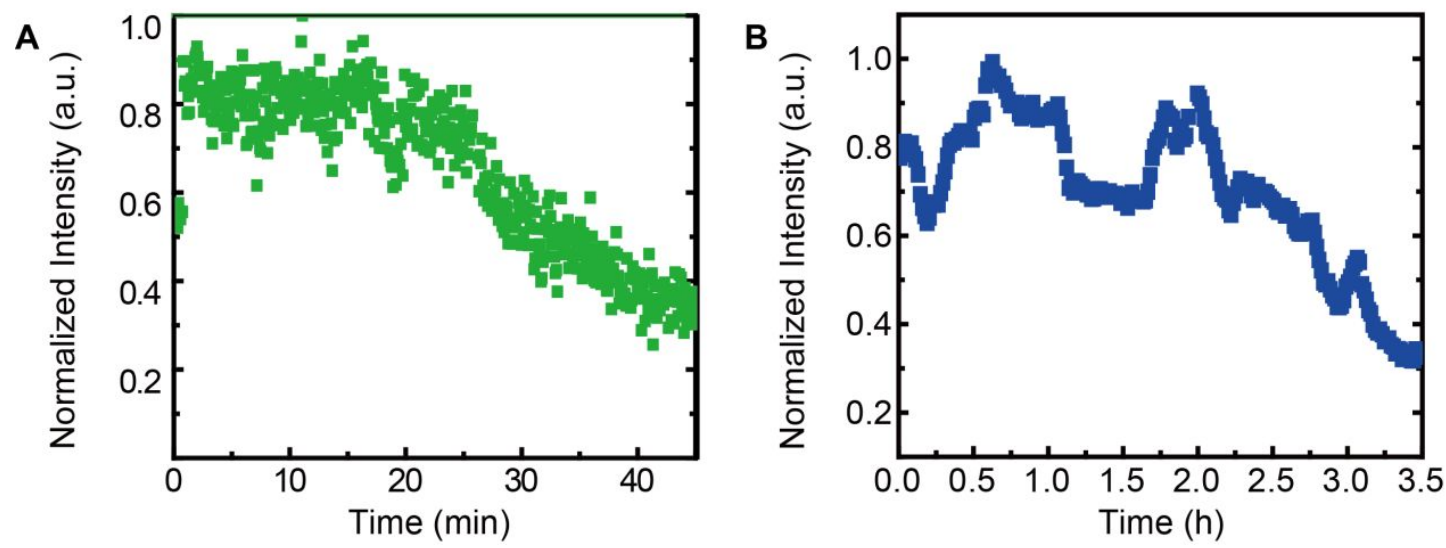
Figure S9. The SERL lifetime at room temperature under atmospheric condition. (a) The laser lifetime of the device without PMMA covered above the perovskite film is about 20-25 min. (b) The stability has been improved to around 3 hours with PMMA coating. The fluctuations are caused by the instability of pumping source.

The lasing photostability has been measured. The device was measured at room temperature under atmospheric condition. The pump intensity was about $\mathrm{P}=1.2 \mathrm{P}_{\text {th }}$ using $400 \mathrm{~nm}$ femtosecond laser. The intensity of the SERL reduced to $1 / \mathrm{e}$ after 30 minutes. Interestingly, the photostability can be extended to 3 hours by simply coating a $200 \mathrm{~nm}$ PMMA layer onto the perovskite film before it is clamped with another DBR mirror.

\section{The spatial coherence of the SERL in the main manuscript}

A
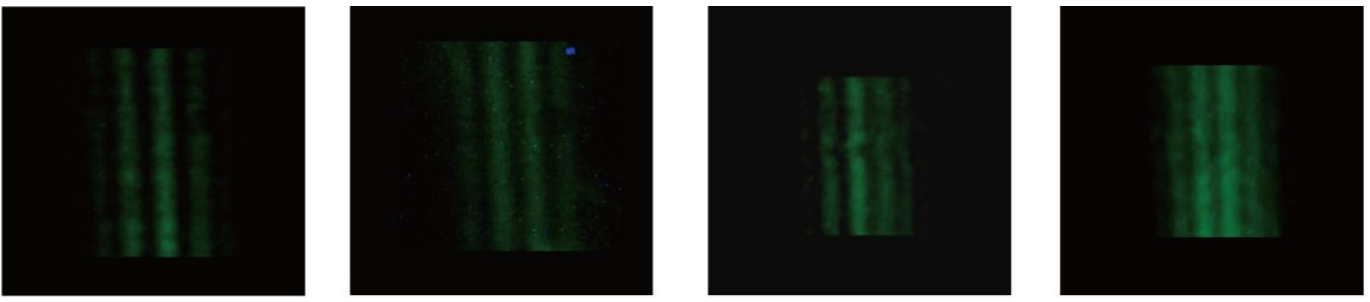

B
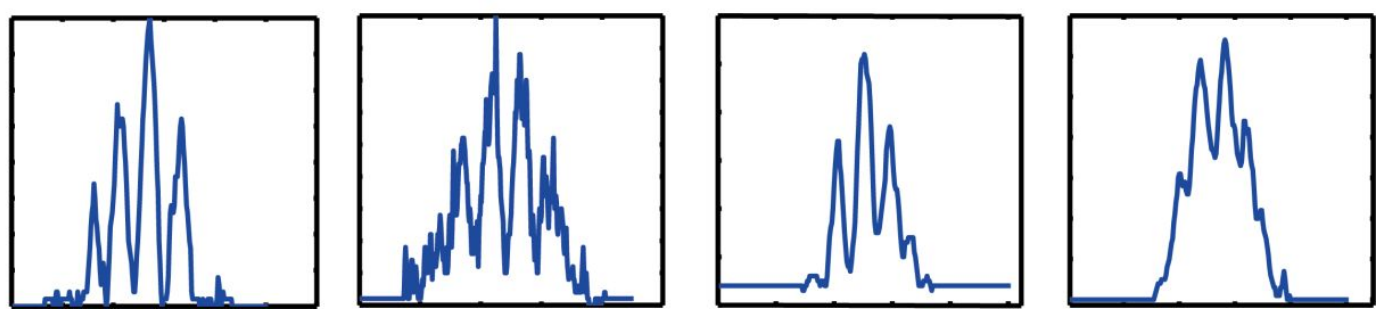

Figure S10. The measurement of spatial coherence at different pump positions. (A)

The interference patterns at different pump positions. (B) The corresponding 
interference patterns.

In the main manuscript, we have mentioned that the spatial coherence of the SERL can also be controlled by changing the pumping position. Here we show the experimental details about the measurements. Similar to the measurement in section-3, here the spatial coherence is also measured by the double-slit interference. All the results are shown in Fig. S10. With the increase of random laser mode number, we can see that the interference pattern becomes more blur. The corresponding intensity distributions in Fig. 10b show that the spatial coherence keeps decreasing from left to right, consistent with the change in the emission spectrum in the main manuscript.

\section{The temporal coherence of the SERL}


A

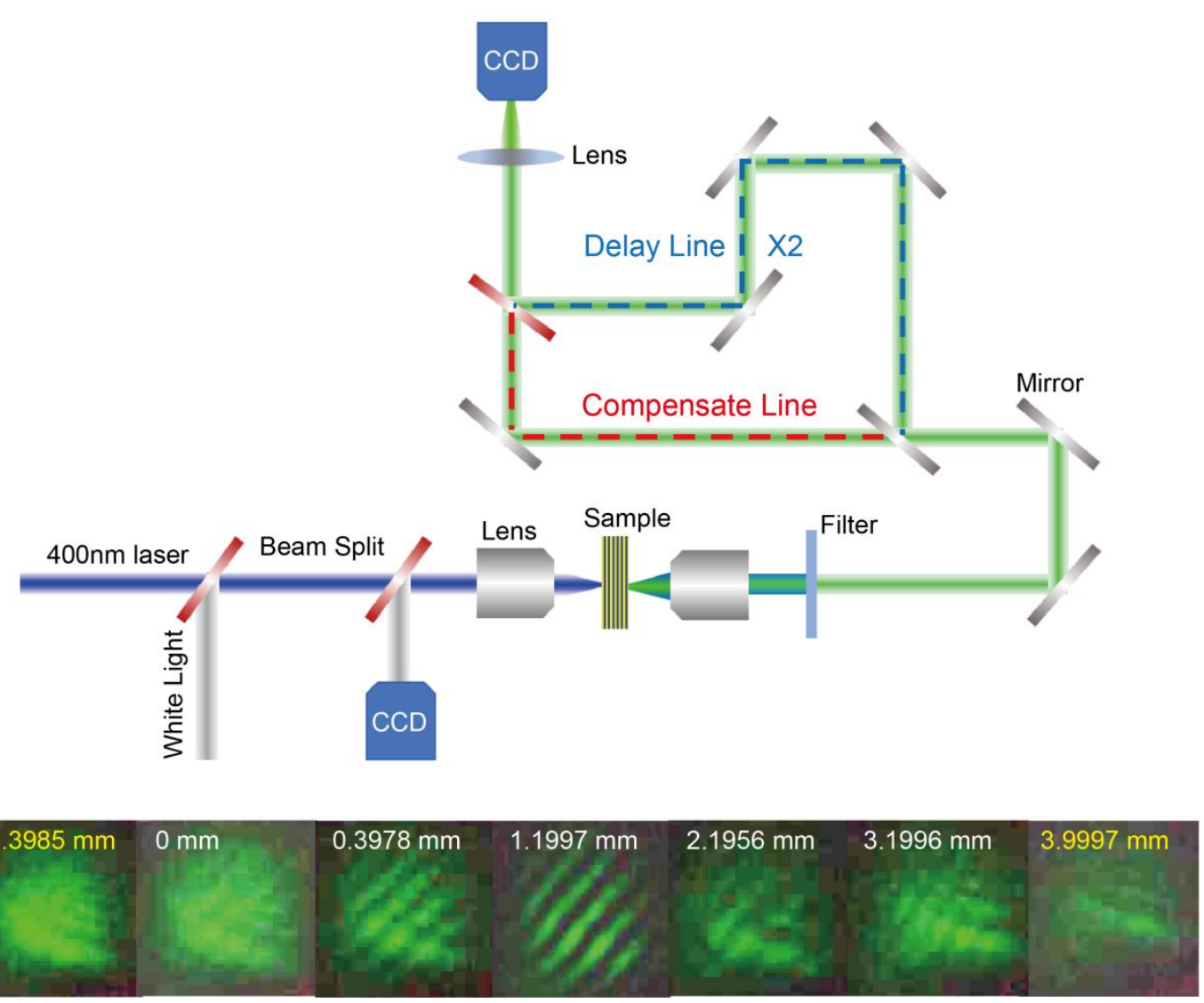

Figure S11. The experiment results of temporal coherence. (A) Optical set up for self-interference measurement of vortex laser emission. (B) The interference fringe of the SERL and corresponding position of the mirror in delay line.

The details of self-interference optical setup are shown in Fig. S11(A). A $400 \mathrm{~nm}$ femtosecond laser as mentioned in part 2 was used to excite the sample. Then the pump light was focused on the surface of SERLs by a 5x objective lens. The emitted light was collected by a 20x object lens. Then, the emitted laser beams were splitted into two beams (50:50) to form a modified Mach-Zehnder interferometer. These two beams passed through different paths called delay line and compensate line and reached the same observation plane. The interference pattern was collected by a CCD. In the experiment, the delay line would uniform scanned in order to ensure the 
temporal overlap between two paths. The position of the moving mirror has been recorded as Fig. S11(B). The distance from the interference fringe appear to vanish is defined as the coherence distance. The coherence distance of the SERLs is about $6.3992 \mathrm{~mm}$, and the coherence time is $21.3307 \mathrm{ps}$. 